\title{
LOCAL LINEAR APPROXIMATIONS OF JUMP DIFFUSION PROCESSES
}

\author{
J. C. JIMENEZ*** AND \\ F. CARBONELL, ${ }^{* * * *}$ Instituto de Cibernética, Matemática y Física
}

\begin{abstract}
Local linear approximations have been the main component in the construction of a class of effective numerical integrators and inference methods for diffusion processes. In this note, two local linear approximations of jump diffusion processes are introduced as a generalization of the usual ones. Their rate of uniform strong convergence is also studied.
\end{abstract}

Keywords: Jump diffusion process; stochastic differential equation; numerical integration; local linearization method; pseudo-likelihood method

2000 Mathematics Subject Classification: Primary 60J75; 60J60; 60H35

\section{Introduction}

In the last ten years, the jump diffusion processes defined through stochastic differential equations (SDEs) have become an important mathematical tool for describing the dynamics of several phenomena, e.g. the dynamics of asset prices in the market and the firing of neurons. Since exact representation of the trajectories of these processes is only possible in a few cases, approximate representations are required. Different types of such approximation have already been proposed in [5], [6], and [7], and are essentially based on Itô-Taylor expansions of the jump diffusion process. The good convergence properties of the approximations based on these expansions and their numerical instability in many cases of nonlinear SDE [1], [8], [9] are well known.

Our main objective in this paper is to investigate another kind of approximation of the jump diffusion processes, namely local linear approximations. In the framework of 'pure' diffusion processes, this kind of approximation has been recently proposed as a stable alternative to the above-mentioned conventional approximations based on Itô-Taylor expansions [1], [3], [9], [12] and they have been the key in the derivation of effective inference methods for SDEs [10], [13], [14], [15]. Therefore, the present study is well motivated.

Specifically, in this note the local linear approximations for diffusion processes are extended to the more general case of jump diffusion processes, and their rate of uniform strong convergence is studied.

Received 23 March 2005; revision received 28 September 2005.

* Postal address: Departamento de Sistemas Adaptativos, Instituto de Cibernética, Matemática y Física, Calle 15, No. 551, Vedado, La Habana 4, C.P. 10400, Cuba.

** Email address: jcarlos@icmf.inf.cu

*** Email address: felix@icmf.inf.cu

Partially supported by the research grant 03-059 RG/MATHS/LA from the Third World Academy of Science. 


\section{Local linear approximations}

Let $(\Omega, \mathcal{F}, \mathrm{P})$ be the underlying complete probability space we consider, and let $\left\{\mathcal{F}_{t}, t \geq t_{0}\right\}$ be an increasing, right-continuous family of complete $\sigma$-subalgebras of $\mathcal{F}$. Consider a $d$-dimensional jump diffusion process $\boldsymbol{x}$ defined by the following SDE:

$$
\begin{aligned}
\mathrm{d} \boldsymbol{x}(t) & =\boldsymbol{f}(t, \boldsymbol{x}(t)) \mathrm{d} t+\sum_{i=1}^{m} \boldsymbol{g}_{i}(t) \mathrm{d} w^{i}(t)+\sum_{i=1}^{p} \boldsymbol{h}_{i}(t, \boldsymbol{x}(t)) \mathrm{d} q^{i}(t) \quad \text { for } t \geq t_{0}, \\
\boldsymbol{x}\left(t_{0}\right) & =\boldsymbol{x}_{0} .
\end{aligned}
$$

Here $\boldsymbol{f}, \boldsymbol{g}_{i}$, and $\boldsymbol{h}_{i}$ are differentiable functions, $\boldsymbol{w}=\left(w^{1}, \ldots, w^{m}\right)$ is an $m$-dimensional $\mathcal{F}_{t^{-}}$ adapted standard Wiener process, and each $q^{i}(t)$ could be either an $\mathscr{F}_{t}$-adapted Poisson counting process $n^{i}(t)$ with intensity $\mu^{i}$, or an $\mathcal{F}_{t}$-adapted compensated Poisson process $n^{i}(t)-\mu^{i} t$. It is assumed that $w^{i}(t)$ and $q^{j}(t)$ are all independent, with zero probability of there being simultaneous jumps. Linear growth restriction, uniform Lipschitz, and smoothness conditions are assumed of the functions $\boldsymbol{f}, \boldsymbol{g}_{i}$, and $\boldsymbol{h}_{i}$ in order to ensure the existence of a unique strong solution to (1).

Consider the time discretization $(\tau)_{\delta}=\left\{\tau_{n}, n=0,1, \ldots\right\}$, with maximum step size $\delta \in$ $(0,1)$, defined as a sequence of $\mathcal{F}$-stopping times that satisfy

$$
t_{0}=\tau_{0}<\cdots<\tau_{n}<\cdots<\infty \text { and } \sup _{n}\left(\tau_{n+1}-\tau_{n}\right) \leq \delta
$$

with probability 1 , where $\tau_{n}$ is $\mathcal{F}_{\tau_{n}}$-measurable for each $n \in \mathbb{N}$. In addition, let us write

$$
n_{t}=\max \left\{n=0,1, \ldots: \tau_{n} \leq t\right\}<\infty
$$

for all $t \in \mathbb{R}$.

In the next subsection, the basic definitions of the local linear approximations of diffusion processes are briefly presented.

\subsection{Local linear approximations of diffusion processes}

Let us consider the $d$-dimensional diffusion process $z$ defined by the SDE

$$
\begin{aligned}
\mathrm{d} z(t) & =\overline{\boldsymbol{f}}(t, \boldsymbol{z}(t)) \mathrm{d} t+\boldsymbol{G}(t) \mathrm{d} \boldsymbol{w}(t) \quad \text { for } t \geq a, \\
\boldsymbol{z}(a) & =z_{0},
\end{aligned}
$$

where $\overline{\boldsymbol{f}}$ is a differentiable function and $\boldsymbol{G}(t)=\left[\boldsymbol{g}_{1}(t), \ldots, \boldsymbol{g}_{i}(t)\right]$ is a $d \times m$ matrix. Here $\boldsymbol{g}_{i}$ and $\boldsymbol{w}$ are defined as before.

Definition 1. ([3].) For a given time discretization $(\tau)_{\delta}$, the order- $\gamma$ local linear discretization $(\gamma=1,1.5)$ of the diffusion process $z$ is defined by the recurrent relation

$$
\boldsymbol{y}_{\tau_{n+1}}=\boldsymbol{y}_{\tau_{n}}+\boldsymbol{F}_{\gamma}\left(\tau_{n}, \boldsymbol{y}_{\tau_{n}} ; \tau_{n+1}-\tau_{n}\right)+\boldsymbol{\xi}\left(\tau_{n}, \boldsymbol{y}_{\tau_{n}} ; \tau_{n+1}-\tau_{n}\right),
$$

where $\boldsymbol{y}_{\tau_{0}}$ is a given initial point and $\boldsymbol{F}_{\gamma}$ and $\boldsymbol{\xi}$ are vector functions defined as

$$
\begin{aligned}
\boldsymbol{\xi}(t, \boldsymbol{y} ; h) & =\int_{t}^{t+h} \exp (\boldsymbol{J}(t, \boldsymbol{y})(t+h-s)) \boldsymbol{G}(s) \mathrm{d} \boldsymbol{w}(s), \\
\boldsymbol{F}_{\gamma}(t, \boldsymbol{y} ; h) & =\int_{0}^{h} \exp (\boldsymbol{J}(t, \boldsymbol{y}) s) \boldsymbol{\phi}_{\gamma}(h-s ; t, \boldsymbol{y}) \mathrm{d} s .
\end{aligned}
$$


Here $\boldsymbol{J}(t, \boldsymbol{y})$ is the Jacobian matrix of $\overline{\boldsymbol{f}}$ evaluated at $(t, \boldsymbol{y}) \in \mathbb{R} \times \mathbb{R}^{d}$, and

$$
\boldsymbol{\phi}_{\gamma}(s ; t, \boldsymbol{y})= \begin{cases}\overline{\boldsymbol{f}}(t, \boldsymbol{y})+\left(\frac{\partial \overline{\boldsymbol{f}}(t, \boldsymbol{y})}{\partial t}+\frac{1}{2} \sum_{k, l=1}^{d}\left[\boldsymbol{G}(t) \boldsymbol{G}^{\top}(t)\right]^{k, l} \frac{\partial^{2} \overline{\boldsymbol{f}}(t, \boldsymbol{y})}{\partial y^{k} \partial y^{l}}\right) s, & \gamma=1.5, \\ \overline{\boldsymbol{f}}(t, \boldsymbol{y})+\frac{\partial \overline{\boldsymbol{f}}(t, \boldsymbol{y})}{\partial t} s, & \gamma=1 .\end{cases}
$$

On the basis of this discretization, various local linearization schemes for the numerical integration of SDEs have been proposed [1], [2], [4], [8], that differ in how the integrals (4) and (5) are computed.

Definition 2. ([3].) For a given time discretization $(\tau)_{\delta}$, the stochastic process

$$
\boldsymbol{y}_{\gamma}^{\delta}=\left\{\boldsymbol{y}_{\gamma}^{\delta}(t), t \in[a, b]\right\}
$$

is called the order- $\gamma$ local linear approximation $(\gamma=1,1.5)$ of the diffusion process $z$ if

$$
\boldsymbol{y}_{\gamma}^{\delta}(t)=\boldsymbol{y}_{\tau_{n_{t}}}+\boldsymbol{F}_{\gamma}\left(\tau_{n_{t}}, \boldsymbol{y}_{\tau_{n_{t}}} ; t-\tau_{n_{t}}\right)+\boldsymbol{\xi}\left(\tau_{n_{t}}, \boldsymbol{y}_{\tau_{n_{t}}} ; t-\tau_{n_{t}}\right),
$$

where $\boldsymbol{y}_{\tau_{n_{t}}}$ is the order- $\gamma$ local linear discretization (3).

It is obvious that the local linear approximation (6) is a continuous-time stochastic process that coincides with the local linear discretization (3) on the time discretization $(\tau)_{\delta}$. In [3], some properties of such an approximation were studied, including its convergence rate to the underlying diffusion process.

\subsection{Local linear approximations of jump diffusion processes}

Consider the sequence of jump times $\{\sigma\}_{\mu^{i}}=\left\{\sigma_{i, n}, n=0,1,2, \ldots\right\}$, associated to $q^{i}(t)$, which is defined as an increasing sequence of random variables such that $\sigma_{i, n+1}-\sigma_{i, n}$ is exponentially distributed with parameter $\mu^{i}$ for all $n$ and $i$. Without loss of generality, it is assumed that $\{\sigma\}_{\mu^{i}} \subset(\tau)_{\delta}$ for all $i=1, \ldots, p$. In addition, let us assume that only the first $r$ Poisson processes $q^{i}$ are compensated, and let $(\tau)_{\delta} \subset\left[t_{0}, T\right]$, with $T<\infty$.

It is well known [11] that the solution to (1) is given by

$$
\boldsymbol{x}(t)=\boldsymbol{x}(t-)+\sum_{i=1}^{p} \boldsymbol{h}_{i}(\boldsymbol{t}, \boldsymbol{x}(t-)) \Delta n_{t}^{i},
$$

where $\Delta n_{t}^{i}$ is the increment of the process $n^{i}$ at the time instant $t$ and $\boldsymbol{x}(t-)$ denotes the solution to (2) with

$$
\overline{\boldsymbol{f}}(t, \boldsymbol{z}(t))=\boldsymbol{f}(t, \boldsymbol{z}(t))-\sum_{j=1}^{r} \boldsymbol{h}_{j}(t, \boldsymbol{z}(t)) \boldsymbol{\mu}^{j} t
$$

and initial condition $\boldsymbol{z}\left(\sigma_{i, n}\right)=\boldsymbol{x}\left(\sigma_{i, n}\right)$, for all times $t$ between two consecutive jump times $\sigma_{i, n}$ and $\sigma_{j, m}$.

This leads to the following two definitions.

Definition 3. For a given time discretization $(\tau)_{\delta}$, the order- $\gamma$ local linear discretization $(\gamma=$ $1,1.5)$ of the jump diffusion process $\boldsymbol{x}$ is defined by the recurrent relation

$$
\boldsymbol{y}_{\tau_{n+1}}=\boldsymbol{y}_{\tau_{n+1}-}+\sum_{i=1}^{p} \boldsymbol{h}_{i}\left(\boldsymbol{\tau}_{n+1}, \boldsymbol{y}_{\tau_{n+1}-}\right) \Delta n_{\tau_{n+1}}^{i},
$$

where $\boldsymbol{y}_{\tau_{n+1}-}$ denotes the local linear discretization of diffusion process $\boldsymbol{z}$ at time $\tau_{n+1}$. 
Definition 4. For a given time discretization $(\tau)_{\delta}$, the stochastic process

$$
\boldsymbol{y}_{\gamma}^{\delta}=\left\{\boldsymbol{y}_{\gamma}^{\delta}(t), t \in\left[t_{0}, T\right]\right\}
$$

is called the order- $\gamma$ local linear approximation $(\gamma=1,1.5)$ of the jump diffusion process $\boldsymbol{x}$ if

$$
\boldsymbol{y}_{\gamma}^{\delta}(t)=\boldsymbol{y}_{\gamma}^{\delta}(t-)+\sum_{i=1}^{p} \boldsymbol{h}_{i}\left(t, \boldsymbol{y}_{\gamma}^{\delta}(t-)\right) \Delta n_{t}^{i},
$$

where $\boldsymbol{y}_{\gamma}^{\delta}(t-)$ denotes the local linear approximation of diffusion process $\boldsymbol{z}$ at time $t$.

\section{Convergence of the local linear approximations}

In order to study the rate of convergence of the above local linear approximations, other definitions (from [7]) are needed.

Let $\mathcal{M}$ be the set of all the multi-indices $\alpha=\left(j_{1}, \ldots, j_{l(\alpha)}\right)$ with $j_{i} \in\{0,1, \ldots, m\}$ and $i=1, \ldots, l(\alpha)$. By $l(\alpha)$ we denote the length of the multi-index $\alpha$ and by $n(\alpha)$ the number of its zero components. The multi-indices in $\mathcal{M}$ obtained by deleting the first and the last components of $\alpha$ are denoted by $-\alpha$ and $\alpha-$, respectively. The multi-index of zero length is denoted by $v$.

Denote by $\mathcal{A}_{\gamma}$ the hierarchical set defined as

$$
\mathcal{A}_{\gamma}=\left\{\alpha \in \mathcal{M}: l(\alpha)+n(\alpha) \leq 2 \gamma \text { or } l(\alpha)=n(\alpha)=\gamma+\frac{1}{2}\right\}
$$

and by $\mathscr{B}\left(\mathcal{A}_{\gamma}\right)=\left\{\alpha \in \mathcal{M} \backslash \mathcal{A}_{\gamma}:-\alpha \in \mathcal{A}_{\gamma}\right\}$ the remainder set of $\mathcal{A}_{\gamma}$, for $\gamma=1$, 1.5. Denote by $H_{\alpha}$ the set of adapted right-continuous processes $h=\left\{h(t), t \geq t_{0}\right\}$ with left limits, on $(\Omega, \mathcal{F}, \mathrm{P})$, such that $\left\{I_{\alpha-}[h(\cdot)]_{\rho, t}, t \geq t_{0}\right\} \in H_{\left(j_{l(\alpha)}\right)}$, where $I_{\alpha-}[h]_{\rho, t}$ denotes the multiple Itô integral of $h$ corresponding to the multi-index $\alpha-$, evaluated at the times $\rho$ and $t$. Here, $H_{(j)}=H_{(1)}$ for $j=\{2, \ldots, m\}$, and $H_{(1)}, H_{(0)}$, and $H_{v}$ are the sets of processes $h$ such that, respectively, $\int_{0}^{t}|h(s, \varpi)|^{2} \mathrm{~d} s<\infty, \int_{0}^{t}|h(s, \varpi)| \mathrm{d} s<\infty$, and $|h(t, \varpi)|<\infty$ hold with probability 1 (with $\varpi \in \Omega$ ).

Furthermore, let

$$
L^{0}=\frac{\partial}{\partial t}+\sum_{k=1}^{d} \bar{f}^{k} \frac{\partial}{\partial x^{k}}+\frac{1}{2} \sum_{k, l=1}^{d} \sum_{j=1}^{m} G^{k, j} G^{l, j} \frac{\partial^{2}}{\partial x^{k} \partial x^{l}}
$$

be the diffusion operator for the SDE (2), let

$$
L^{j}=\sum_{k=1}^{d} G^{k, j} \frac{\partial}{\partial x^{k}} \quad \text { for } j=1, \ldots, m,
$$

and let the vector $\boldsymbol{G}^{j}$ be the $j$ th column of $\boldsymbol{G}$. Denote by $C^{1,2}$ the space of functions from $\mathbb{R} \times \mathbb{R}^{d}$ to $\mathbb{R}^{d}$ that are once and twice continuously differentiable in their first and second arguments, respectively.

The following lemma presents a known result on the boundedness and convergence rate of the local linear approximation (6) for SDEs. It shall be useful to demonstrate an analogous result for the local linear approximation (10) in the case of SDEs with jumps. 
Lemma 1. ([3, Lemma 8].) Suppose that the Itô coefficient functions $\lambda_{\alpha}$, defined for each multi-index $\alpha=\left(j_{1}, \ldots, j_{l}\right)$ by

$$
\lambda_{\alpha}= \begin{cases}L^{j_{1}} \cdots L^{j_{l-1}} \overline{\boldsymbol{f}}, & j_{l}=0 \\ L^{j_{1}} \cdots L^{j_{l-1}} \boldsymbol{G}^{j_{l}}, & j_{l} \neq 0\end{cases}
$$

satisfy $\left|\lambda_{\alpha}(t, \boldsymbol{u})-\lambda_{\alpha}(t, \boldsymbol{v})\right| \leq K_{1}|\boldsymbol{u}-\boldsymbol{v}|$ for all $\alpha \in \mathcal{A}_{\gamma}, t \in[a, b]$, and $\boldsymbol{u}, \boldsymbol{v} \in \mathbb{R}^{d} ; \lambda_{-\alpha} \in C^{1,2}$ and $\lambda_{\alpha} \in H_{\alpha}$ for all $\alpha \in \mathcal{A}_{\gamma} \cup \mathcal{B}\left(\mathcal{A}_{\gamma}\right)$; and $\left|\lambda_{\alpha}(t, \boldsymbol{u})\right| \leq K_{1}(1+|\boldsymbol{u}|)$ for all $\alpha \in \mathcal{A}_{\gamma} \cup \mathscr{B}\left(\mathcal{A}_{\gamma}\right)$, $t \in[a, b]$, and $\boldsymbol{u} \in \mathbb{R}^{d}$. In addition, suppose that the inequality $\left|\boldsymbol{\phi}_{\gamma}(\zeta ; s, \boldsymbol{u})\right| \leq K_{2}$ holds for all $\zeta \in[0,1], s \in[a, b]$, and $\boldsymbol{u} \in \mathbb{R}^{d}$. Then the order- $\gamma$ local linear approximation (6) of the solution to (2) satisfies

$$
\mathrm{E}\left(\sup _{a \leq t \leq b}\left|\boldsymbol{y}_{\gamma}^{\delta}(t)\right|^{2} \mid \mathcal{F}_{a}\right) \leq K_{3}\left(1+\left|\boldsymbol{y}_{\gamma}^{\delta}(a)\right|^{2}\right)
$$

and

$$
\mathrm{E}\left(\sup _{a \leq t \leq b}\left|z(t)-\boldsymbol{y}_{\gamma}^{\delta}(t)\right|^{2} \mid \mathcal{F}_{a}\right) \leq K_{4}\left|z_{0}-\boldsymbol{y}_{\gamma}^{\delta}(a)\right|^{2}+\left(K_{5}\left(1+\left|z_{0}\right|^{2}\right)+K_{3}\left(1+\left|\boldsymbol{y}_{\gamma}^{\delta}(a)\right|^{2}\right)\right) \delta^{2 \gamma},
$$

for $b<\infty$ and $\gamma=1,1.5$. Here $K_{1}, K_{2}, K_{3}, K_{4}$, and $K_{5}$ are positive constants.

The next lemma gives an upper bound for the second moment of the local linear approximation of the jump diffusion process. A key step in the proof is the successive application of (11) in each time interval between two consecutive jumps.

Lemma 2. Let $\overline{\boldsymbol{f}}$ be defined as in (8), and assume that the functions $\overline{\boldsymbol{f}}$ and $\boldsymbol{G}^{j_{l}}$ satisfy the conditions of Lemma 1 for $t \in\left[t_{0}, T\right]$. In addition, suppose that the inequality

$$
\left|\boldsymbol{h}_{i}(t, \boldsymbol{u})\right| \leq K_{6}(1+|\boldsymbol{u}|)
$$

holds for $t \in\left[t_{0}, T\right]$ and $\boldsymbol{u} \in \mathbb{R}^{d}$. Then the order- $\gamma$ local linear approximation (10) of the solution to (1) satisfies

$$
\mathrm{E}\left(\sup _{t_{0} \leq s \leq T}\left|\boldsymbol{y}_{\gamma}^{\delta}(s)\right|^{2} \mid \mathcal{F}_{t_{0}}\right) \leq D\left(1+\left|\boldsymbol{y}_{\gamma}^{\delta}\left(t_{0}\right)\right|^{2}\right),
$$

where $D$ is a positive constant.

Proof. Let $N_{T}=\sum_{i=1}^{p} n^{i}(T)$ be the total number of jumps up to time $T$, and let

$$
\{t\}_{N_{T}}=\left\{t_{j}, j=0, \ldots, N_{T}\right\}
$$

be a sequence of times such that $\{t\}_{N_{T}} \subset(\tau)_{\delta}, t_{j} \in\left\{\tau_{0} \cup\{\sigma\}_{\mu^{1}} \cup \cdots \cup\{\sigma\}_{\mu^{p}}\right\}$, and $t_{j}<t_{j+1}$, for all $j=0, \ldots, N_{T}-1$. Furthermore, let

$$
Z_{s}=\left\{n^{i}\left(t_{j}\right): t_{j} \leq s, t_{j} \in\{t\}_{N_{T}}, i=1, \ldots, p\right\}
$$

for $s \geq t_{0}$, and define

$$
e_{j}=\mathrm{E}\left(\sup _{t_{0} \leq s \leq t_{j}}\left|\boldsymbol{y}_{\gamma}^{\delta}(s)\right|^{2} \mid \mathcal{F}_{t_{0}}, Z_{t_{j}}\right)
$$


with $t_{j} \in\{t\}_{N_{T}}$; here, $\mathrm{E}\left(\cdot \mid \mathcal{F}_{t_{0}}, Z_{t_{j}}\right)$ denotes the conditional expectation with respect to $\mathcal{F}_{t_{0}}$ and $Z_{t_{j}}$. Then

$$
e_{j+1} \leq e_{j}+\Delta e_{j+1},
$$

where $\Delta e_{j+1}=\mathrm{E}\left(\sup _{t_{j}<s \leq t}\left|\boldsymbol{y}_{\gamma}^{\delta}(s)\right|^{2} \mid \mathcal{F}_{t_{0}}, Z_{t_{j+1}}\right)$. From (10) and (13) we find that

$$
\begin{aligned}
\Delta e_{j+1} \leq & 2 \mathrm{E}\left(\sup _{t_{j}<s \leq t_{j+1}}\left|\boldsymbol{y}_{\gamma}^{\delta}(s-)\right|^{2} \mid \mathcal{F}_{t_{0}}, Z_{t_{j+1}}\right) \\
& +2 p \sum_{i=1}^{p} \mathrm{E}\left(\sup _{t_{j}<s \leq t_{j+1}}\left|\boldsymbol{h}_{i}\left(s, \boldsymbol{y}_{\gamma}^{\delta}(s-)\right) \Delta n_{s}^{i}\right|^{2} \mid \mathcal{F}_{t_{0}}, Z_{t_{j+1}}\right) \\
\leq & 2\left(1+2 p K_{6}^{2}\right) \mathrm{E}\left(\sup _{t_{j} \leq s \leq t_{j+1}}\left|\boldsymbol{y}_{\gamma}^{\delta}(s-)\right|^{2} \mid \mathcal{F}_{t_{0}}, Z_{t_{j+1}}\right)+4 p K_{6}^{2} .
\end{aligned}
$$

By definition, for all $s \in\left[t_{j}, t_{j+1}\right], \boldsymbol{y}_{\gamma}^{\delta}(s-)$ is the local linear approximation of the solution to an SDE (with no jumps). Therefore, by using Lemma 1 in this time interval, we find that

$$
\begin{aligned}
\mathrm{E}\left(\sup _{t_{j} \leq s \leq t_{j+1}}\left|\boldsymbol{y}_{\gamma}^{\delta}(s-)\right|^{2} \mid \mathcal{F}_{t_{0}}, Z_{t_{j+1}}\right) & =\mathrm{E}\left(\mathrm{E}\left(\sup _{t_{j} \leq s \leq t_{j+1}}\left|\boldsymbol{y}_{\gamma}^{\delta}(s-)\right|^{2} \mid \mathcal{F}_{t_{j}}\right) \mid \mathcal{F}_{t_{0}}, Z_{t_{j+1}}\right) \\
& \leq K_{3}\left(1+\mathrm{E}\left(\left|\boldsymbol{y}_{\gamma}^{\delta}\left(t_{j}\right)\right|^{2} \mid \mathcal{F}_{t_{0}}, Z_{t_{j+1}}\right)\right) \\
& \leq K_{3}\left(1+\mathrm{E}\left(\sup _{t_{0} \leq s \leq t_{j}}\left|\boldsymbol{y}_{\gamma}^{\delta}\left(t_{j}\right)\right|^{2} \mid \mathcal{F}_{t_{0}}, Z_{t_{j}}\right)\right) .
\end{aligned}
$$

Thus,

$$
\Delta e_{j+1} \leq C_{1} e_{j}+C_{2},
$$

where $C_{1}=2\left(1+2 p K_{6}^{2}\right) K_{3}$ and $C_{2}=C_{1}+4 p K_{6}^{2}$, and it follows that

$$
e_{j+1} \leq\left(1+C_{1}\right) e_{j}+C_{2}
$$

which implies that

$$
\begin{aligned}
e_{j+1} & \leq\left(1+C_{1}\right)^{j+1} e_{0}+\frac{C_{2}}{C_{1}}\left(\left(1+C_{1}\right)^{j}-1\right) \\
& \leq\left(1+C_{1}\right)^{j+1}\left(e_{0}+\frac{C_{2}}{C_{1}}\right) \\
& \leq \frac{C_{2}}{C_{1}}\left(1+C_{1}\right)^{j+1}\left(1+e_{0}\right) .
\end{aligned}
$$

By using the above inequality and taking $j=N_{T}$, we find that

$$
\mathrm{E}\left(\sup _{t_{0} \leq s \leq T}\left|\boldsymbol{y}_{\gamma}^{\delta}(s)\right|^{2} \mid \mathcal{F}_{t_{0}}, Z_{t_{N_{T}}}\right) \leq \frac{C_{2}}{C_{1}}\left(1+C_{1}\right)^{1+N_{T}}\left(1+\left|\boldsymbol{y}_{\gamma}^{\delta}\left(t_{0}\right)\right|^{2}\right) .
$$

Finally, by taking into account the fact that

$$
\mathrm{E}\left(\kappa^{N_{T}}\right)=\mathrm{E}\left(\mathrm{e}^{N_{T} \log (\kappa)}\right)=\exp \left((\kappa-1)\left(T-t_{0}\right) \sum_{i=1}^{p} \mu^{i}\right)
$$


for all constant $\kappa>1$, we obtain

$$
\begin{aligned}
\mathrm{E}\left(\sup _{t_{0} \leq s \leq T}\left|\boldsymbol{y}_{\gamma}^{\delta}(s)\right|^{2} \mid \mathcal{F}_{t_{0}}\right) & =\mathrm{E}\left(\mathrm{E}\left(\sup _{t_{0} \leq s \leq T}\left|\boldsymbol{y}_{\gamma}^{\delta}(s)\right|^{2} \mid \mathcal{F}_{t_{0}}, Z_{t_{N_{T}}}\right) \mid \mathcal{F}_{t_{0}}\right) \\
& \leq \frac{C_{2}}{C_{1}}\left(1+C_{1}\right) \exp \left(C_{1}\left(T-t_{0}\right) \sum_{i=1}^{p} \mu^{i}\right)\left(1+\left|\boldsymbol{y}_{\gamma}^{\delta}\left(t_{0}\right)\right|^{2}\right)
\end{aligned}
$$

which completes the proof.

The following theorem states the convergence rate of the local linear approximation of the jump diffusion process. A key step in the proof is the successive application of (12) in each time interval between two consecutive jumps.

Theorem 1. Let $\overline{\boldsymbol{f}}$ be defined as in (8), and suppose that the functions $\overline{\boldsymbol{f}}$ and $\boldsymbol{G}^{j_{l}}$ satisfy the conditions of Lemma 1 for $t \in\left[t_{0}, T\right]$. In addition, suppose that the inequality

$$
\left|\boldsymbol{h}_{i}(t, \boldsymbol{u})-\boldsymbol{h}_{i}(t, \boldsymbol{v})\right| \leq K_{6}|\boldsymbol{u}-\boldsymbol{v}|
$$

holds for $t \in\left[t_{0}, T\right]$ and $\boldsymbol{u}, \boldsymbol{v} \in \mathbb{R}^{d}$. Then the order- $\gamma$ local linear approximation (10) of the solution to (1) satisfies

$$
\begin{aligned}
& \mathrm{E}\left(\sup _{t_{0} \leq t \leq T}\left|\boldsymbol{x}(t)-\boldsymbol{y}_{\gamma}^{\delta}(t)\right|^{2} \mid \mathcal{F}_{t_{0}}\right) \\
& \quad \leq D_{4}\left|\boldsymbol{x}_{0}-\boldsymbol{y}_{\gamma}^{\delta}\left(t_{0}\right)\right|^{2}+\left(D_{5}\left(1+\left|\boldsymbol{x}_{0}\right|^{2}\right)+D_{3}\left(1+\left|\boldsymbol{y}_{\gamma}^{\delta}\left(t_{0}\right)\right|^{2}\right)\right) \delta^{2 \gamma}
\end{aligned}
$$

for $T<\infty$ and $\gamma=1,1.5$. Here $D_{3}, D_{4}$, and $D_{5}$ are positive constants.

Proof. As in the proof of Lemma 2, let $N_{T}=\sum_{i=1}^{p} n^{i}(T)$ be the total number of jumps up to time $T$, and let $\{t\}_{N_{T}}=\left\{t_{j}, j=0, \ldots, N_{T}\right\}$ be a sequence of times such that $\{t\}_{N_{T}} \subset(\tau)_{\delta}$, $t_{j} \in\left\{\tau_{0} \cup\{\sigma\}_{\mu^{1}} \cup \cdots \cup\{\sigma\}_{\mu^{p}}\right\}$, and $t_{j}<t_{j+1}$, for all $j=0, \ldots, N_{T}-1$. Furthermore, let

$$
Z_{s}=\left\{n^{i}\left(t_{j}\right): t_{j} \leq s, t_{j} \in\{t\}_{N_{T}}, i=1, \ldots, p\right\}
$$

for $s \geq t_{0}$, and define

$$
e_{j}=\mathrm{E}\left(\sup _{t_{0} \leq t \leq t_{j}}\left|\boldsymbol{x}(t)-\boldsymbol{y}_{\gamma}^{\delta}(t)\right|^{2} \mid \mathcal{F}_{t_{0}}, Z_{t_{j}}\right),
$$

with $t_{j} \in\{t\}_{N_{T}}$. In this case, we have $e_{j+1} \leq e_{j}+\Delta e_{j+1}$, where

$$
\Delta e_{j+1}=\mathrm{E}\left(\sup _{t_{j}<t \leq t_{j+1}}\left|\boldsymbol{x}(t)-\boldsymbol{y}_{\gamma}^{\delta}(t)\right|^{2} \mid \mathcal{F}_{t_{0}}, Z_{t_{j+1}}\right) .
$$

By using (7), (10), and (17) we find that

$$
\begin{aligned}
\Delta e_{j+1} \leq & 2 \mathrm{E}\left(\sup _{t_{j}<t \leq t_{j+1}}\left|\boldsymbol{x}(t-)-\boldsymbol{y}_{\gamma}^{\delta}(t-)\right|^{2} \mid \mathcal{F}_{t_{0}}, Z_{t_{j+1}}\right) \\
& +2 p \sum_{i=1}^{p} \mathrm{E}\left(\sup _{t_{j}<t \leq t_{j+1}}\left|\boldsymbol{h}_{i}(t, \boldsymbol{x}(t-)) \Delta n_{t}^{i}-\boldsymbol{h}_{i}\left(t, \boldsymbol{y}_{\gamma}^{\delta}(t-)\right) \Delta n_{t}^{i}\right|^{2} \mid \mathcal{F}_{t_{0}}, Z_{t_{j+1}}\right) \\
\leq & D_{1} \mathrm{E}\left(\sup _{t_{j}<t \leq t_{j+1}}\left|\boldsymbol{x}(t-)-\boldsymbol{y}_{\gamma}^{\delta}(t-)\right|^{2} \mid \mathcal{F}_{t_{0}}, Z_{t_{j+1}}\right),
\end{aligned}
$$


where $D_{1}=2\left(1+p K_{6}^{2}\right)$. By definition, for all $t \in\left[t_{j}, t_{j+1}\right], \boldsymbol{y}_{\gamma}^{\delta}(t-)$ is the local linear approximation of the solution to an SDE (with no jumps). Therefore, by using Lemma 1 in this time interval, we find that

$$
\begin{aligned}
R:= & \mathrm{E}\left(\sup _{t_{j}<t \leq t_{j+1}}\left|\boldsymbol{x}(t-)-\boldsymbol{y}_{\gamma}^{\delta}(t-)\right|^{2} \mid \mathcal{F}_{t_{0}}, Z_{t_{j+1}}\right) \\
\leq & \mathrm{E}\left(\mathrm{E}\left(\sup _{t_{j} \leq t \leq t_{j+1}}\left|\boldsymbol{x}(t-)-\boldsymbol{y}_{\gamma}^{\delta}(t-)\right|^{2} \mid \mathcal{F}_{t_{j}}\right) \mid \mathcal{F}_{t_{0}}, Z_{t_{j+1}}\right) \\
\leq & K_{4} \mathrm{E}\left(\left|\boldsymbol{x}\left(t_{j}\right)-\boldsymbol{y}_{\gamma}^{\delta}\left(t_{j}\right)\right|^{2} \mid \mathcal{F}_{t_{0}}, Z_{t_{j+1}}\right) \\
& +\left(K_{5}\left(1+\mathrm{E}\left(\left|\boldsymbol{x}\left(t_{j}\right)\right|^{2} \mid \mathcal{F}_{t_{0}}, Z_{t_{j+1}}\right)\right)+K_{3}\left(1+\mathrm{E}\left(\left|\boldsymbol{y}_{\gamma}^{\delta}\left(t_{j}\right)\right|^{2} \mid \mathscr{F}_{t_{0}}, Z_{t_{j+1}}\right)\right)\right) \delta^{2 \gamma} .
\end{aligned}
$$

From inequalities (14) and (15) it follows that

$$
\begin{aligned}
\mathrm{E}\left(\left|\boldsymbol{y}_{\gamma}^{\delta}\left(t_{j}\right)\right|^{2} \mid \mathcal{F}_{t_{0}}, Z_{t_{j+1}}\right) & \leq \mathrm{E}\left(\sup _{t_{0} \leq t \leq t_{j}}\left|\boldsymbol{y}_{\gamma}^{\delta}(t)\right|^{2} \mid \mathcal{F}_{t_{0}}, Z_{t_{j}}\right) \\
& \leq \mathrm{E}\left(\sup _{t_{0} \leq t \leq T}\left|\boldsymbol{y}_{\gamma}^{\delta}(t)\right|^{2} \mid \mathcal{F}_{t_{0}}, Z_{t_{N_{T}}}\right) \\
& \leq \frac{C_{2}}{C_{1}}\left(1+C_{1}\right)^{1+N_{T}}\left(1+\left|\boldsymbol{y}_{\gamma}^{\delta}\left(t_{0}\right)\right|^{2}\right)
\end{aligned}
$$

The inequality

$$
\begin{aligned}
\mathrm{E}\left(\left|\boldsymbol{x}\left(t_{j}\right)\right|^{2} \mid \mathcal{F}_{t_{0}}, Z_{t_{j+1}}\right) & \leq \mathrm{E}\left(\sup _{t_{0} \leq t \leq T}|\boldsymbol{x}(t)|^{2} \mid \mathcal{F}_{t_{0}}, Z_{t_{N_{T}}}\right) \\
& \leq \frac{C_{2}^{*}}{C_{1}^{*}}\left(1+C_{1}^{*}\right)^{1+N_{T}}\left(1+\left|\boldsymbol{x}\left(t_{0}\right)\right|^{2}\right)
\end{aligned}
$$

can be analogously derived for the jump diffusion process $\boldsymbol{x}$ by following the proof of Lemma 2, using the fact that the well-known inequality

$$
\mathrm{E}\left(\sup _{t_{0} \leq t \leq T}|z(t)|^{2} \mid \mathcal{F}_{t_{0}}\right) \leq K_{5}\left(1+\left|z\left(t_{0}\right)\right|^{2}\right),
$$

rather than (11), holds for the solution to (2). In (19), $C_{1}^{*}=2\left(1+2 p K_{6}^{2}\right) K_{5}$ and $C_{2}^{*}=$ $C_{1}^{*}+4 p K_{6}^{2}$ are positive constants. However, we also have

$$
\mathrm{E}\left(\left|\boldsymbol{x}\left(t_{j}\right)-\boldsymbol{y}_{\gamma}^{\delta}\left(t_{j}\right)\right|^{2} \mid \mathcal{F}_{t_{0}}, Z_{t_{j+1}}\right) \leq \mathrm{E}\left(\sup _{t_{0} \leq t \leq t_{j}}\left|\boldsymbol{x}\left(t_{j}\right)-\boldsymbol{y}_{\gamma}^{\delta}\left(t_{j}\right)\right|^{2} \mid \mathcal{F}_{t_{0}}, Z_{t_{j}}\right) .
$$

From (18), (19), and (20) it follows that

$$
R \leq K_{4} e_{j}+D_{2} \delta^{2 \gamma}
$$

where

$$
\begin{aligned}
& D_{2}=2 \alpha \beta^{N_{T}+1}\left(K_{5}\left(1+\left|\boldsymbol{x}\left(t_{0}\right)\right|^{2}\right)+K_{3}\left(1+\left|\boldsymbol{y}_{\gamma}^{\delta}\left(t_{0}\right)\right|^{2}\right)\right), \\
& \alpha=\max \left\{\frac{C_{2}}{C_{1}}, \frac{C_{2}^{*}}{C_{1}^{*}}\right\}, \quad \beta=\max \left\{\left(1+C_{1}\right),\left(1+C_{1}^{*}\right)\right\} .
\end{aligned}
$$


Thus,

$$
\Delta e_{j+1} \leq D_{1}\left(K_{4} e_{j}+D_{2} \delta^{2 \gamma}\right)
$$

and it follows that

$$
e_{j+1} \leq\left(1+D_{1} K_{4}\right) e_{j}+D_{1} D_{2} \delta^{2 \gamma}
$$

which implies that

$$
\begin{aligned}
e_{j+1} & \leq\left(1+D_{1} K_{4}\right)^{j+1} e_{0}+\frac{D_{2} \delta^{2 \gamma}}{K_{4}}\left(\left(1+D_{1} K_{4}\right)^{j}-1\right) \\
& \leq\left(1+D_{1} K_{4}\right)^{j+1}\left(e_{0}+\frac{D_{2} \delta^{2 \gamma}}{K_{4}}\right)
\end{aligned}
$$

and, therefore,

$$
e_{N_{T}+1} \leq\left(1+D_{1} K_{4}\right)^{N_{T}+1}\left(e_{0}+\frac{D_{2} \delta^{2 \gamma}}{K_{4}}\right) .
$$

By taking into account expression (21) for $D_{2}$, we have

$$
e_{N_{T}+1} \leq\left(1+D_{1} K_{4}\right)^{N_{T}+1} e_{0}+\frac{2 \alpha \rho^{N_{T}+1}\left(K_{5}\left(1+\left|\boldsymbol{x}\left(t_{0}\right)\right|^{2}\right)+K_{3}\left(1+\left|\boldsymbol{y}_{\gamma}^{\delta}\left(t_{0}\right)\right|^{2}\right)\right)}{K_{4}} \delta^{2 \gamma},
$$

where $\rho=\beta\left(1+D_{1} K_{4}\right)$. That is,

$$
\begin{aligned}
& \mathrm{E}\left(\sup _{t_{0} \leq t \leq T}\left|\boldsymbol{x}(t)-\boldsymbol{y}_{\gamma}^{\delta}(t)\right|^{2} \mid \mathcal{F}_{t_{0}}, Z_{t_{N_{T}}}\right) \\
& \quad \leq C_{4}\left|\boldsymbol{x}\left(t_{0}\right)-\boldsymbol{y}_{\gamma}^{\delta}\left(t_{0}\right)\right|^{2}+\left(C_{5}\left(1+\left|\boldsymbol{x}\left(t_{0}\right)\right|^{2}\right)+C_{3}\left(1+\left|\boldsymbol{y}_{\gamma}^{\delta}\left(t_{0}\right)\right|^{2}\right)\right) \delta^{2 \gamma},
\end{aligned}
$$

where $C_{4}=\left(1+D_{1} K_{4}\right)^{N_{T}+1}, C_{5}=2 \alpha \rho^{N_{T}+1} K_{5} / K_{4}$, and $C_{3}=2 \alpha \rho^{N_{T}+1} K_{3} / K_{4}$.

Finally, by using (16) we obtain

$$
\begin{aligned}
\mathrm{E}\left(\sup _{t_{0} \leq t \leq T}\left|\boldsymbol{x}(t)-\boldsymbol{y}_{\gamma}^{\delta}(t)\right|^{2} \mid \mathcal{F}_{t_{0}}\right)= & \mathrm{E}\left(\mathrm{E}\left(\sup _{t_{0} \leq t \leq T}\left|\boldsymbol{x}(t)-\boldsymbol{y}_{\gamma}^{\delta}(t)\right|^{2} \mid \mathcal{F}_{t_{0}}, Z_{t_{N_{T}}}\right) \mid \mathscr{F}_{t_{0}}\right) \\
\leq & D_{4}\left|\boldsymbol{x}\left(t_{0}\right)-\boldsymbol{y}_{\gamma}^{\delta}\left(t_{0}\right)\right|^{2}+\left\{D_{5}\left(1+\left|\boldsymbol{x}\left(t_{0}\right)\right|^{2}\right)\right. \\
& +D_{3}\left(1+\left|\boldsymbol{y}_{\gamma}^{\delta}\left(t_{0}\right)\right|^{2}\right\} \delta^{2 \gamma},
\end{aligned}
$$

where $D_{4}=\left(1+D_{1} K_{4}\right) \mathrm{e}^{\varepsilon D_{1} K_{4}}, D_{5}=2 \alpha \rho \mathrm{e}^{\varepsilon(\rho-1)} K_{5} / K_{4}, D_{3}=2 \alpha \rho \mathrm{e}^{\varepsilon(\rho-1)} K_{3} / K_{4}$, and $\varepsilon=\left(T-t_{0}\right) \sum_{i=1}^{p} \mu^{i}$ are positive constants. This completes the proof.

Finally, by using the Lyapunov inequality the following result is straightforward to derive.

Corollary 1. In addition to the assumptions of Theorem 1, suppose that the initial conditions satisfy

$$
\mathrm{E}\left(\left|\boldsymbol{x}_{0}\right|^{2}\right)<\infty, \quad \mathrm{E}\left(\left|\boldsymbol{y}_{\gamma}^{\delta}\left(t_{0}\right)\right|^{2}\right)<\infty, \quad \mathrm{E}\left(\left|\boldsymbol{x}_{0}-\boldsymbol{y}_{\gamma}^{\delta}\left(t_{0}\right)\right|^{2}\right) \leq K_{7} \delta^{2 \gamma},
$$

where $K_{7}$ is a positive constant. Then the order- $\gamma$ local linear approximation (10) of the solution to (1) satisfies

$$
\mathrm{E}\left(\sup _{t_{0} \leq t \leq T}\left|\boldsymbol{x}(t)-\boldsymbol{y}_{\gamma}^{\delta}(t)\right|\right)=O\left(\delta^{\gamma}\right) .
$$

From a practical point of view, the results above state the uniform order of strong convergence of the local linearization schemes that could be obtained by approximating the integrals (4) and (5) involved in the definition of $\boldsymbol{y}_{\tau_{n+1}}$ in (9), provided that these approximations have the same order of convergence. 


\section{References}

[1] Biscay, R., Jimenez, J. C., Riera, J. and Valdes, P. (1996). Local linearization method for the numerical solution of stochastic differential equations. Ann. Inst. Statist. Math. 48, 631-644.

[2] Jimenez, J. C. (2002). A simple algebraic expression to evaluate the local linearization schemes for stochastic differential equations. Appl. Math. Lett. 15, 775-780.

[3] Jimenez, J. C. And Biscay, R. (2002). Approximation of continuous time stochastic processes by the local linearization method revisited. Stoch. Anal. Appl. 20, 105-121.

[4] Jimenez, J. C., ShoJi, I. AND OzAKI, T. (1999). Simulation of stochastic differential equations through the local linearization method. A comparative study. J. Statist. Phys. 94, 587-602.

[5] LiU, X. Q. AND Li, C. W. (1999). Product expansion for stochastic jump diffusions and its application to numerical approximation. J. Comput. Appl. Math. 108, 1-17.

[6] Maghsoodi, Y. (1998). Exact solutions and doubly efficient approximations of jump-diffusion Itô equations. Stoch. Anal. Appl. 16, 1049-1072.

[7] Mikulevičius, R. and Platen, E. (1988). Time discrete Taylor approximations for Itô processes with jump component. Math. Nachr. 138, 93-104.

[8] OzaKi, T. (1985). Nonlinear time series models and dynamical systems. In Time Series in the Time Domain (Handbook Statist. 5), eds E. J. Hannan et al., North-Holland, Amsterdam, pp. 25-83.

[9] OZAKI, T. (1992). A bridge between nonlinear time series models and nonlinear stochastic dynamical systems: a local linearization approach. Statistica Sinica 2, 113-135.

[10] Prakasa-Rao, B. L. S. (1999). Statistical Inference for Diffussion Type Processes. Oxford University Press.

[11] Protter, P. (1990). Stochastic Integration and Differential Equations. Springer, Berlin.

[12] Schurz, H. (2002). Numerical analysis of stochastic differential equations without tears. In Handbook of Stochastic Analysis and Applications (Statist. Textbook Monogr. 163), eds D. Kannan and V. Lakahmikamtham, Marcel Dekker, New York, pp. 237-359.

[13] ShoJi, I. and OzaKi, T. (1997). Comparative study of estimation methods for continuous time stochastic processes. J. Time Series Anal. 18, 485-506.

[14] ShoJI, I. AND OzaKi, T. (1998). A statistical method of estimation and simulation for systems of stochastic differential equations. Biometrika 85, 240-243.

[15] ShoJI, I. AND OzaKi, T. (1998). Estimation for nonlinear stochastic differential equations by a local linearization method. Stoch. Anal. Appl. 16, 733-752. 